\title{
Describing Community Understanding of Male Involvement in Antenatal, Delivery and Postnatal care; "rural context of Ibanda District, Southwest Uganda"
}

Fred Bagenda ( $\sim$ bagendaf@gmail.com )

Mbarara University of Science and Technology

Vincent Batwala

Mbarara University of Science and Technology

Charles Muchunguzi

Mbarara University of Science and Technology

Jerome Kabakyenga

Mbarara University of Science and Technology

Lynn Atuyambe

Makerere University

\section{Research Article}

Keywords: Describe, male involvement, antenatal, skilled delivery, postnatal care

Posted Date: March 10th, 2021

DOI: https://doi.org/10.21203/rs.3.rs-251336/v1

License: (c) (1) This work is licensed under a Creative Commons Attribution 4.0 International License.

Read Full License 


\section{Abstract}

Introduction:Male involvement in the reproductive health of their partners has been shown to have positive outcomes of health. However male involvement description varies within communities due to different social and gender contexts. The description of male involvement is not documented in Ibanda.Therefore this study was done to describe male involvement in the local context.

Methods:The study was conceptualized, planned, and executed with combined cross-sectional, exploratory, and Grounded Theory research designs because of its complexity in nature and the fact that this theory conceptualizes behavior. Data collection was using qualitative methods; narrative interviews and analyzed using a content-based approach. All methods were done in accordance with the relevant guidelines and regulations.

Results:The two broad themes that describe male involvement were mostly instrumentalist and included the Level of involvement and social support perspective of involvement. Sub-themes were obtained under the description of male involvement as the extent to which males were involved, including just impregnating the wife without further attachment throughout the engagement. The subthemes/activities on the describing of male involvement as the perspective of social support included emotional support, role play in the wellbeing of mother and child, men's preparedness, and physical support. Physical support included; nutritional support, buying clothes for the expected baby, providing transportation, escorting the mother to a health facility, helping the wife with household chores as well as informative/appraisal supports.

Conclusion:The description of male involvement in antenatal, delivery, and postnatal care was mostly based on the "Instrumental" approach with two broad themes of Level of involvement and Social support. The level of involvement varied from none to high while social support included; emotional, having a role to play in the wellbeing of the mother and baby, the preparedness of man, and physical support.It is therefore important to understand the context of male involvement in each community to use the most appropriate intervention to increase male involvement.

\section{Introduction}

Reproductive health is supposed to encompass both men and women for it to be meaningful and holistic. The International Conferenceson Population and Development in Cairo and Beijing of 1994 and 1995 respectively reemphasized the need to increase male partner involvement since they are the Gate Way to maternal and newborn health and better care-seeking. According to Kwambai, et. al,[1] men's reproductive health and their behavior may impact positively and/or negatively on women's reproductive health outcomes and children's well-being in society. Research shows that men not only act as "gatekeepers", restricting women and children in health care seeking but if involved may lead to better healthcareseeking behavior and better utilization of ANC, delivery, and postnatal care services $[1,2,3,4,5,6$ \&25].Male partner involvement includes: encouraging men to become more involved and supportive of 
women's needs, choices, and rights in sexual and reproductive health and addressing men's own sexual and reproductive health needs and behavior $[1,7,8$ \& 9].It is known that male involvement in antenatal, delivery and postnatal improves utilization and uptake of the antenatal, delivery, and postnatal services and this ultimately improves maternal and newborn outcomes $[2,10,11,12,13,21,26,27 \& 28]$. The Ministry of Health in Uganda implements a policy of attending to mothers who come for ANC with a male partner first as a strategy to encourage male partner involvement in ANC, In some district in central and southwestern Uganda the Ministry of Health and partners have some radio spots which encourage men to be involved in ANC, delivery and postnatal care for their partners [14].

Male partner involvement has been described by WHO in various ways; using two main approaches which are the 'gender equity' and the 'Instrumental' approach. The gender equity approach adopts more equitable gender roles such as joint planning and decision-making among couples and shared control of household tasks including parenting. This is expected to enable healthier behaviors and improved healthcare-seeking by the couple [15]. The second approach sees male involvement as more instrumental; the direct and varying assistance provided by men to improve their partners' and children's health through the ANC, delivery, and postnatal periods. This approach considers assistance the man provides irrespective of his gender role [15]. The direct assistance may include; providing emotional support that includes; intimacy, providing financial support, providing transportation to the health facility, attending ANC and or delivery and PNC, helping with household work, providing nutritious food, being receptive to health education information, and providing clothing for the newborn among others. These assistances vary in importance depending on the community's context of gender, education level, nature of employment and the socio-cultural setting of communities, and their understanding of male involvement. Therefore every community has variations as to the description of male involvement $[2,16$, $17,18,25,26,29 \& 30]$. The difference is that the gender equity approach examines its potential to deal with gender inequities that contribute to poor health and the instrumental approach sees the behavior (such as attending ANC) as an end in itself. It is not easy to practically measure male involvement in a way that captures both the ways that men can challenge gender norms and the practical assistance provided by men to women during pregnancy, delivery, and the postnatal period. There are no definitive indicators to measure male involvement and different authors have used a combination of them or a single one to capture male involvement in a broader view $[15,19,20,21,23,26,29,30,31,32$ \& 33].

The male involvement in maternal and newborn health strategy was by the Uganda Ministry of Health. Despite this, the utilization of maternal and reproductive health services in Uganda is still low. This may be attributed to the unclear definition and understanding of male involvement in the local context of Ibanda in Uganda and probably the approach of its implementation. This description of male involvement in reproductive health care is not documented and there are gaps in the way it isunderstood in Ibanda and the local Ugandan context [2, 18 \& 31]. To design interventions that are suitable for the particular Ibanda district context the community understanding of male involvement was described by this study.This will enable us to appreciate the difference and assess the involvement more objectively.The study aimed to investigate community understanding of male involvementin antenatal, delivery, and postnatal care and describe it in the local context of Ibanda district, Uganda. 


\section{Methods}

The study was carried out in the Ibanda district situated in southwestern Uganda The district is bordered by Kamwenge in the north and northwest, Kiruhura district in the east, Buhweju, and Mbarara in the south, and Rubirizi district in the west. It has the predominant tropical vegetation and climate with some hilly and mountainous areas. It consists of 11 sub-counties, 4 town councils, 54 parishes, and 577 villages. The total population is 206,905 of which 112,590 (54.4\%) are female and $11,346(5 \%)$ women expected to be pregnant. The population growth rate is $2.54 \%$ per annum which is slightly lower than the national average of 3.035. The larger proportions of the population are farmers and live in the rural area with the smaller proportion living in the four semi-urban town councils.

\section{Study design}

The study was conceptualized, planned, and executed with combined cross-sectional, exploratory, and Grounded Theory research designs because of its complexity in nature. It also conceptualizes patterns of people's behavior to resolve a concern.

The data collection was mainly through narrative interviews (18) supplemented with direct observation to capture none verbal communication and other behavioral dynamics this was done till saturation was achieved.The tools were narrative interview guides and an observation checklist.

The guides were administered to; pregnant females, men with pregnant women, community health workers, unmarried males, elderly females, elderly males, health workers, Village Health Team members, and local leaders. The recorded qualitative data from the cross-sectional study were transcribed verbatim, read and re-read, coded, and sorted into categories.

The analysis was done using the content-based approach for qualitative data and the themes for describing male involvement in reproductive health were identified [24].

The methods were done according to the relevant guidelines and regulations .

The concept and Proposal of the study were approved by the Higher Degree Research Ethics Committee of Makerere University School of Public Health and the Uganda National Council for Science and Technology as is mandatory. Written permission was got from Ibanda local district authority and individual informed consent was obtained from participants at the time of enrollment into the qualitative study.

\section{Results}

The respondents included nine male and nine female who were purposively selected and these were; married (4 male), unmarried (2 male), pregnant (4), previously pregnant women (2), village health team members ( 3 ; one female, two male) and health workers ( 3 ; one male, two female). 
The two broad themes that describe male involvement were mostly instrumentalist and included the Level of involvement and social support perspective of involvement. Sub-themes were obtained under the description of male involvement as the extent to which males were involved, including just impregnating the wife without further attachment and throughout the engagement. The subthemes/activities on the describing of male involvement as the perspective of social support included emotional support, role play in the wellbeing of mother and child, men's preparedness, and physical support. Physical support included; nutritional support, buying clothes for the expected baby, providing transportation, escorting the mother to a health facility, helping the wife with household chores as well as informative/appraisal support. The specific support activities were prioritized differently by the respondents.

\section{Level/Extent of male involvement}

Study findings show that male involvement is described by the level or extent of male involvement.The study findings provide that where the male involvement was high the pregnant mother had a healthier pregnancy, better delivery outcomes, and a healthy postnatal period. The more involved the male partner the baby is likely to be healthier and the less the male involvement the health of the mother and baby is likely to be poor.

"Of course different people understand it differently but I understand male involvement as the range from no involvement to excellent involvement." Respondent 2 (male)

"All men don't take care of their wives, some do while others don't." Respondent11 (male)

"We have had situations where some men give facilities to the woman but abandon them in other situations, you find the man is touched right from the time he learns that the wife is pregnant and he becomes happy." Respondent 18 (female)

"For my wife even the children we have now when she is pregnant, we walk together to the hospital for antenatal care." Respondent 13(male)

There was another description of male involvement as unbothered male partners are merely interested in having sex and stop at impregnating their wives. The uninterested involvement was usually associated with poor health for the mother and the baby as compared to the contrary.

"There are some men that just go to bars without doing anything." Respondent 9 (female)

\section{Social support}

\section{Emotional support}




\section{Findings describe male involvement as emotional and psychological support to the pregnant mother from her partner.}

Participants revealed that where there was emotional/love and or psychological support from the male partner the pregnancy, delivery and postnatal periods were likely to be healthy.

"In this community, men are different but the biggest percentage of men take care of their wives when they are pregnant because they know that giving birth is hard to even after giving birth, they give us comfort," Respondent 6 (female)

"The man should not also stress her so that the baby can be healthy." Respondent 10 (male)

"Taking care of a woman when she's pregnant, during delivery, and after she has given birth." Respondent 4 (male)

"However, some men do not care for their wives:For our community, most of the men don't put in much effort to take care of their wives when they are pregnant and most times women just take care of themselves." Respondent 11 (male)

\section{Roleplay in the wellbeing of mother and child}

Male involvement was described as the male partner having a role to play during pregnancy, delivery, and postnatal periods.

Participants revealed that if men played their role including among others planning, providing money, love, and care the baby and mother would be healthy.

"Male involvement is a man having roles to play towards healthy child and the mother, it is having a man participate in the wellbeing of the mother and the child that is during pregnancy, labor and after delivery." Respondent 9 (female)

"I understand it that if a woman is pregnant and you take good care of her, it helps both of you to produce a healthy baby and it also helps the mother to stay healthy." Respondent 11(male)

\section{Men's preparedness}

Male involvement is described as men being prepared for the outcome from the time of conception through delivery and postnatal to take care of the pregnant woman and the newborn baby.

Participants thought that if the couple was prepared and planned then the pregnancy, delivery, postnatal, and newborn baby were likely to be better. 
"First of all you find out whether your wife is pregnant and make preparation to take your wife for antenatal checkups." Respondent 3 (male)

"What I know is you have to prepare yourself if she gets pregnant," Respondent 13 (male)

\section{Physical support}

"According to me; I think husbands should take care of mothers, providing what to eat, providing money for health care and whatever they need." Respondent 15 (female)

\section{Nutritional Support to the Pregnant Mothers}

The study finding reveals that male involvement is described as providing nutritional support to pregnant mothers during antenatal, delivery, and postnatal periods.

Study participants felt that a well-fed mother will have good energy during pregnancy and good health will be a crucial support factor during delivery.

Good nutrition throughout the period from conception to post-delivery was associated with better outcomes for both the mother and the newborn.

"I understand it in a way that a man has to bring food that has vitamins and proteins for the pregnant wife so that when it gets to the time of delivery, she can have a normal birth." Respondent 1 (male)

"Taking care of a woman when she's pregnant, during delivery after she has given birth, she should eat vegetables, have a balanced diet." Respondent 4 (male)

"After giving birth, a man should try and take care of me and provide food for me so that I can have a healthylife." Respondent 5 (female)

Buying clothes for the expected baby

The study findings describe male involvement as buying clothes and necessities for the expectant baby in time.

Participants felt that if the man bought the clothes and necessities for the baby the pregnant mothers were happy and felt loved and cared for.

"The man can also buy baby clothes before the baby is born, even if he doesn't have enough money it shows that he cares." Respondent 5 (female)

"I made sure I got all requirements like clothes for the baby, buying what was required at the hospital." Respondent 3 (male) 
"Prepare baby clothes which will be used to carry in the baby at birth, for example, I have already bought baby clothes because am almost giving birth." Respondent 6 (female)

\section{Providing transportation}

Male involvement is understood as providing transport to access antenatal, delivery, and postnatal services.

Participants revealed that when the male partner provided transport whether physical or delegated made the investigation and management of the pregnancy, delivery, and postnatal better.

Findings indicate that when transport was provided any possible complications were detected early and hence a likely better outcome for the baby and mother.

"They even transport us back home, that is if you get any complications like having a cesarean section or others and if you give birth from a nearby hospital like Nyamarebe. They pick you from the hospital and take you back where they can take good care of you." Respondent 6 (female)

"Some have means of transport, they transport their wives themselves and those who don't have, look for motorcycles to bring their wives to the health facilities." Respondent 9 (female)

"Involving is taking care of women by taking her to the hospital for antenatal." Respondent 16 (female)

\section{Escorting wife to the health facility}

The study findings describe male involvement as escorting your wife to the health facility for antenatal, delivery, and or postnatal care.

Participants felt that if the male partner escorted the pregnant women then they would appreciate better the issues surrounding the management of the pregnancy.

The findings however also point out that escorting a wife is not the most important part of male involvement.

"Men try to take their wives for antenatal care and at delivery time." Respondent 7 (male)

"The husband takes care that is; escorts her to the hospital for antenatal and we as health workers when these pregnant women are brought by their husbands, we care about them by giving them a priority." Respondent 8 (female)

"Some physically come with their wives during delivery and they become attendants for these mothers who have given birth." Respondent 9 (female) 
"Men think that men's involvement in antenatal is just escorting your wife to the hospital because when we talk to them, they show you, they wanted to come." Respondent 18 (female)

\section{Helping wife with household chores}

Male involvement was described partially as helping the pregnant or postnatal woman, especially with the heavy household chores.

Participants felt that the pregnant and postnatal mother was weak and needed rest so if they were helped with household chores the mother and baby would be healthy. The mother also felt loved and cared for and this had a positive relationship with the male partner and the newborn baby.

"Other situations, you find the man is touched right from the time he learns that the wife is pregnant, he becomes happy, helps the wife in house chores." Respondent 2 (male)

"A man should help his wife on some home chores especially those that are heavy." Respondent 18 (female)

\section{Informative and appraisal support}

\section{Taking the HIV test with the mother}

Findings reveal that male involvement is understood as taking the HIV test together with the pregnant mother to prevent transmission of HIV to the newborn baby.

Study participants felt that taking the HIV test was critical since it could enable the health workers to take the necessary intervention to have a healthy HIV-free baby but also for the couple to know their HIV status if they did not.

Findings indicate that if the HIV status was known then the couple would have better trust for each other in the relationship.

"The other thing that we do is to teach them about HIV/AIDs and test them for HIV, so when a couple comes together, it becomes easy for us to disclose the results whether they are positive or negative." Respondent 8 (female)

"Sometimes you might find they have HIV but they are not aware of it, but if they go together for checkups, it would help them give birth to a healthy baby." Respondent 12 (male)

"To me, most of the men don't usually help their wives that is during antenatal on some work at home." Respondent 14(female) 


\section{Discussion}

\section{Level of Involvement}

The level of male partner involvement was one of the criteria for describing this is similar tosome other studies done in Afghanistan [26], Bangladesh [25\& 30],Ethiopia [2, 31 \&33],Ghana [17], India [4], Kenya [16 \& 27], Mozambique [23], South Africa [9] and Tanzania [11\& 32].It was found that the levels of male partner involvement varied from poor to good for antenatal, delivery, and postnatal care with antenatal usually being the highest and postnatal being the lowest for most of the cases. However in all of the above mentioned the level of involvement was not used alone but was combined with one or more of the specific instrumentalist activities like; accompanying spouse for health care, providing physical and financial support, fear of or happy to have HIV test results, saving money for the period of antenatal, delivery and postnatal and male partner attendance of antenatal, delivery and postnatal care, providing emotional support, providing instrumental and informational support, preparedness for the birth and have knowledge of complications, identify the delivery place oridentify skilled birth attendant.In this study, however unlikeRedshaw and Hendersons, theactivities of attending ultrasound and labor or delivery are not among the activities that male partners get involved in but the level of involvement is also part of the description of male involvement by the above mentioned [3].

The level of male involvement is important in describing male involvement and varies in different settings and the parameters used to determine it are many and may be used differently and the individual importance of these parameters are also variable based on the different context of the specific community[2, 11, 14, 16, 17, 18\&21].

\section{Social support}

\section{Emotional support}

Emotional support has been used as one of the social support criteriafor describing male involvement in antenatal and postnatal periods. This is in agreement with work in South Africa [9], the USA [8], and Bangladesh [25]. This criterion was not found to be used alone to describe male involvement and there some contextsin which this criterion is not used to describe male involvement [1, 26 \& 33]

Role to play in the wellbeing of the mother and child is another social support criterion used to describe male involvement through some activities like identifying the place of delivery or skilled birth attendant, the decision on the place of delivery, and joint decision making.This finding is similar to studies in Mozambique [23],Nigeria [34], and Kenya [1] as well as in agreement with the WHO approach of "Gender Equity" [15]. However, some studies did not use this criterion at all as part of their description of male involvement [2, 25 \&27]. 
Preparedness of the man was used under social support to describe male involvement and this is in agreement with Tadese et al [33],Wai et al [29], Singh et al [7], andMullany et al [20]. This criterion is not used alone but in combination with others. However, some studies did not use preparedness as any of their criteria for describing male involvement in antenatal, delivery, and postnatal care $[3,4 \& 29]$. The better-prepared men were likely to have better outcomes for both the mother and the newborn child.

\section{Physical Support}

Nutritional support was one of the most common descriptions of physical support. This finding echoes that earlier reported in Tanzania [11], India [4], and South Africa[9].On the other hand, some studies in Nepal [20], Uganda [7], and Ethiopia [31] did not include nutritional support at all as part of the description of male involvement.

Buying clothes for the expected baby was a physical support activity used to describe male involvementalthough not used alone. This is in agreement with other findings in the USA, Tanzania [11], and Kenya [1] where it was also used. However according to Dadi et al [28], Aguier\&Jenning [21], and Peneza\& Maluku [14] they did not include the activity of buying clothes for newborns. A mother whose newborn baby had clothes bought felt loved and cared for.

Providing transportation was mentioned as either physically transporting or finding transport for the wife to go to the health facility. This is also in agreement with findings in Bangladesh [30], Afghanistan [26], and Kenya [27]. Whileother literature did not use this activity to describe male involvement although there is evidence that this reduces the second delay of the mother moving from home to the health facility [ 9 \& 25].

Escorting wife to and or attendingantenatal, delivery, and or postnatal care at the health facilitywas used as an activity to describe male involvement. This is similar to that reported elsewhere in Ghana [17], Kenya [1\&27], Bangladesh [30], Ethiopia [2, 28\&31], Afghanistan [26], England [3], Uganda [7] and Tanzania [11]. In some of the above, this activity is used alone to describe male involvement in antenatal, delivery, and postnatal care. Escorting the mother to a health facility leads to better dealing with complications and understanding of the condition of the mother and baby.

Helping a wife with household chores especially the heavy work is used to describe male involvement but this is used in combination with other activities and not alone. Kwambai et al [1] and Dutta et al [4] also used this to describe male involvement in combination with other activities. Helping the mother with chores enables her to rest enough and have sufficient energy and good nutritional reserves

Informative and appraisal support was used to define male involvement but also in combination with other activities. Tadese et al [33] and Story et al [25] also used this activity in Ethiopia and Bangladesh respectively. These usually have information about danger signs and other investigations and are a good motivator for the men to be involved in antenatal, delivery, and postnatal care. 


\section{Conclusion And Recommendation}

The description of male involvement in antenatal, delivery, and postnatal care in Ibanda was mostly based on the "Instrumental" approach with two broad themes of the level of involvement and Social support. The level of involvement varied from none to high while social support included; emotional support, having a role to play in the wellbeing of the mother and baby, the preparedness of the man for the period, and physical support. Physical support included; nutritional support, buying clothes for the expected child, providing transportation, escorting wife to the health facility, helping wife with household chores, and informative \& appraisal support. In describing male involvement as a combination of the above activities and varies in different communities. It is therefore important to understand the context of male involvement in each community to use the most appropriate intervention to increase male involvement.

\section{Abbreviations}

ANC; Antenatal Care, HIV; Human Immunodeficiency Virus, Kl; Key Informant, PNC; Postnatal Care and WHO; World Health Organization.

\section{Declarations}

\section{Acknowledgment.}

The School of Public Health, Makerere University. Department of Community Health, Mbarara University of Science and Technology, Ibanda District Health workers, Data collectors, Village Health Teams, and the community members that participated.

\section{Author's contributions}

FB was involved in the conception and design of the study, its implementation, analysis of the data, interpretation of the findings, and drafting of the paper. VB participated in the conception of the study and review of the paper.CM participated in the collection and analysis of data, interpretation of the findings, and review of the paper. JKwas involved in the conception of the study and review of the paper. LA was involved inthe conception and design of the study and review of the paper. All the authors of this manuscript have read and approved it.

\section{Funding}

The funding is by the Swedish International Development Agency (SIDA) as a Ph.D. scholarship through the Makerere-Sweden Bilateral Research Programme. 


\section{Availability of data}

All the data including the audio recording are available with the corresponding author Fred Bagenda, Department of Community Health, Mbarara University of Science and Technology, PO Box 1410, Mbarara, Uganda, email: bagendaf@gmail.com Tel: + 256772452506.

\section{Ethical approval}

The study was approved by the Higher Degree Research Ethics Committee of Makerere University School of Public Health and the Uganda National Council for Science and Technology. Written permission was got from Ibanda local district authority and informed consent from the individual participants.

\section{Competing interests}

The authors declare that there are no competing interests.

\section{References}

1. Kwambai TK, Dellicour S, Desai M, Ameh CA, Person B, Achieng F, Mason L, Laserson KF, TerKuile FO. Perspectives of men on antenatal and delivery care service utilization in rural western Kenya: a qualitative study. BMC Pregnancy \& Childbirth. (2013);13:134. DOI: 10.1186/1471-2393-13-134.

2. Mohammed BH, Johnston JM, Vackova D, Hassen SM, Yi H. The role of the male partner in the utilization of maternal health care services in Ethiopia: a community-based couple study. BMC Pregnancy \& Childbirth. (2019);19(1):28. DOI: 10.1186/s12884-019-2176-z. .

3. Redshaw M, Henderson J. Fathers' engagement in pregnancy and childbirth: evidence from a national survey. BMC Pregnancy Childbirth. (2013):13:70. DOI: 10.1186/1471-2393-13-70.

4. Dutta, M., Kapilashrami, M. C., \& Tiwari, V. K. Knowledge, awareness and extent of male participation in key areas of reproductive and child health in an urban slum of Delhi. Health and Population: Perspectives and Issues:(2004): 27(2), 49-66.

5. Gallen, M., Liskin, L., \&Kak, N.-New Focus for Family Planning Programs. Population Reports: (2002): XIV(52), 1-28.

6. Becker, S., \& Robinson, J. C. Reproductive health care: services oriented to couples. International Journal of Gynaecology and Obstetrics: The:(1998) 61(3), 275-281.

7. Singh, D., Lample, M., \& Earnest, J. The involvement of men in maternal health care: cross-sectional, pilot case studies from Maligita and Kibibi, Uganda. Reproductive Health:(2014):11(1), 68-75.

8. Alio, A. P., Lewis, C. A., Scarborough, K., Harris, K., \&Fiscella, K. A community perspective on the role of fathers during pregnancy: a qualitative study. BMC Pregnancy and Childbirth. (2013):13(1), 60-70. 
9. Maman, S., Moodley, D., \& Groves, A. K. Defining male support during and after pregnancy from the perspective of HIV-positive and HIV-negative women in Durban, South Africa. Journal of Midwifery and Women's Health, (2011) 56(4), 325-331.

10. Alemayehu M, Gebrehiwot TG, Medhanyie AA, Desta A, Alemu T, Abrha A, Godefy H. Utilization and factors associated with antenatal, delivery and postnatal Care Services in Tigray Region, Ethiopia: a community-based cross-sectional study. BMC Pregnancy Childbirth. (2020);20(1):334. DOI: 10.1186/s12884-020-03031-6.

11. August, F., Pembe, A. B., Mpembeni, R., Axemo, P., \&Darj, E. Community health workers can improve male involvement in maternal health: Evidence from rural Tanzania. Global Health Action,(2016). 9(1). https://doi.org/10.3402/gha.v9.30064

12. Darega B, Dida N, Tafese F, Ololo S. Institutional delivery and postnatal care services utilization in AbunaGindeberet District, West Shewa, Oromiya Region, Central Ethiopia: A Community-based crosssectional study. BMC Pregnancy Childbirth. (2016);16:149. DOI: 10.1186/s12884-016-0940-x.

13. Nyondo, A. L., Chimwaza, A. F., \&Muula, A. S. (2014). Exploring the relevance of male involvement in the prevention of mother-to-child transmission of HIV services in Blantyre, Malawi. BMC International Health and Human Rights, 14(1), 30-41.

14. Peneza AK, Maluka SO. 'Unless you come with your partner you will be sent back home': strategies used to promote male involvement in antenatal care in Southern Tanzania. Glob Health Action. (2018);11(1):1449724. DOI: 10.1080/16549716.2018.1449724.

15. World Health Statistics 2015. https://doi.org/10.1017/СB09781107415324.004

16. Kabanga E, Chibwae A, Basinda N, Morona D. Prevalence of male partners involvement in antenatal care visits - in Kyela district, Mbeya. BMC Pregnancy Childbirth. (2019);19(1):321. DOI: 10.1186/s12884-019-2475-4.

17. Craymah JP, Oppong RK, Tuoyire DA. Male Involvement in Maternal Health Care at Anomabo, Central Region, Ghana. Int J Reprod Med. (2017); DOI: 10.1155/2017/2929013.

18. Comrie-Thomson, L., Tokhi, M., Ampt, F., Portela, A., Chersich, M., Khanna, R., \&Luchters, S. Challenging gender inequity through male involvement in maternal and newborn health: critical assessment of an emerging evidence base. Culture, Health, and Sexuality.(2015) 17, S177-S189. https://doi.org/10.1080/13691058.2015.1053412.

19. Byamugisha, R, Tumwine J K, Semiyaga, N, \&Tylleskär. T. Determinants of male involvement in the prevention of mother-to-child transmission of HIV program in Eastern Uganda: a cross-sectional survey. Reproductive Health:(2010): 7, 12.

20. Britta C. Mullany, S Becker, MJ Hindin, The impact of including husbands in antenatal health education services on maternal health practices in urban Nepal: results from a randomized controlled trial, Health Education Research. (2007):Volume 22, Issue 2, , Pages 166-176, https://doi.org/10.1093/her/cyl060.

21. Aguiar C \& Jennings L. Impact of Male partner Antenatal Accompaniment on Perinatal outcomes in Developing Countries: A Systematic Literature Review:(2015):19:2012-2019 DOI 10.1007/s10995- 
015-1713-2

22. Kashitala J, Nyambe N, Mwalo S, Musamba J, Chishinga N, Kasonde P, Lilja AM, Mwiche A, Welsh M. Is Male Involvement in ANC and PMTCT Associated with Increased Facility-Based Obstetric Delivery in Pregnant Women? Afr J Reprod Health. (2015);19(2):117-24.

23. Galle A, De Melo M, Griffin S, Osman N, Roelens K, Degomme O. A cross-sectional study of the role of men and the knowledge of danger signs during pregnancy in southern Mozambique. BMC Pregnancy Childbirth. (2020);20(1):572. DOI: 10.1186/s12884-020-03265-4.

24. Ritchie, J., Lewis, J., Nicholls, C., \&Ormston, R. QUALITATIVE RESEARCH PRACTICE. (2013). London: SAGE.

25. Story, W.T., Burgard, S.A., Lori, J.R. et al. Husbands' involvement in delivery care utilization in rural Bangladesh: A qualitative study. BMC Pregnancy \& Childbirth 12, 28 (2012). https://doi.org/10.1186/1471-2393-12-28

26. Alemi S, Nakamura K, Rahman M, Seino K. Male participation in antenatal care and its influence on their pregnant partners' reproductive health care utilization: insight from the 2015 Afghanistan Demographic and Health Survey. Journal of Biosocial Sci. (2020) 15:1-23. DOI: $10.1017 /$ S0021932020000292

27. Odeny B, McGrath CJ, Langat A, Pintye J, Singa B, Kinuthia J, Katana A, Ng'ang'a L, John-Stewart G. Male partner antenatal clinic attendance is associated with increased uptake of maternal health services and infant BCG immunization: a national survey in Kenya. BMC Pregnancy Childbirth. (2019);19(1):284. DOI: 10.1186/s12884-019-2438-9.

28. Dadi LS, Berhane M, Ahmed Y, Gudina EK, Berhanu T, Kim KH, Getnet M, Abera M. Maternal and newborn health services utilization in Jimma Zone, Southwest Ethiopia: a community based crosssectional study. BMC Pregnancy Childbirth. (2019 );19(1):178. DOI: 10.1186/s12884-019-2335-2

29. Wai KM, Shibanuma A, Oo NN, Fillman TJ, Saw YM, Jimba M. Are Husbands Involving in Their Spouses' Utilization of Maternal Care Services?: A Cross-Sectional Study in Yangon, Myanmar. PLoS One. (2015);10(12):e0144135. DOI: 10.1371/journal.pone.0144135.

30. Rahman AE, Perkins J, Islam S, Siddique AB, Moinuddin M, Anwar MR, Mazumder T, Ansar A, Rahman MM, Raihana S, Capello C, Santarelli C, El Arifeen S, Hoque DME. Knowledge and involvement of husbands in maternal and newborn health in rural Bangladesh. BMC Pregnancy Childbirth. (2018): 18;18(1):247. DOI: 10.1186/s12884-018-1882-2.

31. Teklesilasie W, Deressa W. Husbands' involvement in antenatal care and its association with women's utilization of skilled birth attendants in Sidama zone, Ethiopia: a prospective cohort study. BMC Pregnancy Childbirth. (2018);18(1):315. DOI: 10.1186/s12884-018-1954-3.

32. Gibore NS, Bali TAL, Kibusi SM. Factors influencing men's involvement in antenatal care services: a cross-sectional study in a low resource setting, Central Tanzania. Reprod Health. (2019);16(1):52. DOI: 10.1186/s12978-019-0721-x.

33. Tadesse M, Boltena AT, Asamoah BO. Husbands' participation in birth preparedness and complication readiness and associated factors in WolaitaSodo town, Southern Ethiopia. Afr J Prim 
Health Care Fam Med. (2018);10(1):e1-e8. DOI: 10.4102/phcfm.v10i1.1471

34. Falade-Fatila $O$ and Adebayo A M. Male partners'involvement in pregnancy-related care among married men in Ibadan,Nigeria. Reproductive Health (2020); 17:14. doi.org/10.1186/s12978-0200850-. 